\title{
Aberration of IncRNA LINC00460 is a Promising Prognosis Factor and Associated with Progression of Clear Cell Renal Cell Carcinoma
}

\author{
Shijie Zhang' \\ Fengyun Zhang ${ }^{2}$ \\ Yingdong $\mathrm{Niu}^{3}$ \\ Shenglong $\mathrm{Yu}^{4}$
}

'General Surgery, The Second Affiliated Hospital of Mudanjiang Medical University, Mudanjiang, Heilongjiang, I570I I, People's Republic of China; ${ }^{2}$ Hematology and Rheumatology, The Second Affiliated Hospital of Mudanjiang Medical University, Mudanjiang, Heilongjiang, I570I I, People's Republic of China; ${ }^{3}$ Supply Room, The Second Affiliated Hospital of Mudanjiang Medical University, Mudanjiang, Heilongjiang, I570I I, People's Republic of China; ${ }^{4}$ Urology Surgery, The Second Affiliated Hospital of Mudanjiang Medical University, Mudanjiang, Heilongjiang, I570I I, People's Republic of China
Correspondence: Shenglong Yu Urology Surgery, The Second Affiliated Hospital of Mudanjiang Medical University, No. 15 Dongxiaoyun Street, Aimin District, Mudanjiang, Heilongjiang, I570 I I, People's Republic of China Tel +86-13945395825

Email yusImmu@I63.com
Purpose: Long noncoding RNAs have been studied more and more as potential prognostic markers. However, the prognostic of LINC00460 in clear cell renal cell carcinoma (ccRCC) has not been explored. In this study, the potential role of LINC00460 was investigated in ccRCC.

Patients and Methods: One hundred thirteen pairs of ccRCC tissues and para-normal tissues were collected. The expressions of LINC00460 in these tissues and ccRCC cells were evaluated via qRT-PCR. The prognostic value of LINC00460 was accessed with the use of Kaplan-Meier analysis and Cox proportional hazards model analysis. The influence of LINC00460 on ccRCC cell proliferation, migration, and invasion was determined via cell counting kit-8 (CCK-8) and Transwell assays.

Results: The results revealed that LINC00460 was significantly enhanced in ccRCC tissues, as well as in ccRCC cell lines. The overexpression of LINC00460 was significantly associated with lymph node metastasis and TNM stage, and lead to poor overall survival. Knockdown of LINC00460 reduces the cell ability of proliferation, migration, and invasion. LINC00460 could sponge to miR-149-5p.

Conclusion: LINC00460 may be developed as a prognostic biomarker and molecular therapy target for ccRCC.

Keywords: LINC00460, clear cell renal cell carcinoma, miR-149-5p, prognosis

\section{Introduction}

Renal cell carcinoma (RCC) accounts for $90 \%$ of all kidney neoplasms and has up to seventh place in common cancers among men. ${ }^{1,2}$ In 2016 WHO classification of renal tumors, RCCs are classified into ten types. Among all different histological types, clear cell renal cell carcinoma (ccRCC) with a proportion of $80 \%$ to $90 \%$ were the most in RCC. ${ }^{3}$ There was evidence that about $60 \%$ of the RCC incidences are found in incidental diagnosis. ${ }^{1}$ In addition, ccRCC itself possesses a worse prognosis compared to papillary or chromophobe RCC. ${ }^{4}$ All these demonstrate that there is an urgent need to explore more reliable and easily available biomarkers for predictive and prognostic utility.

Long noncoding RNAs (lncRNAs), with more than 200 transcripts nucleotides in length, have no protein-coding potential functionally. Recently, lncRNAs have been studied more and more as potential prognostic markers, because of their unique expression in respective tissues or particular cancer types and exquisite regulation that can restrict to specific cell types. ${ }^{5-7}$ For instance, it is reported that 
the expression of lnc-PCTST is down-regulated in pancreatic cancer tissues and predicts a poor prognosis. ${ }^{8}$ Even, one kind of long noncoding RNA may be a prognosis biomarker in several tumors concurrently, such as CDKN2B-AS1 (ANRIL) which is a poor prognosis biomarker in intrahepatic cholangiocarcinoma, hepatocellular carcinoma, or uterine corpus endometrial carcinoma. ${ }^{9}$ LINC00460 was reported to be associated with poor prognosis of several cancers, such as lung squamous cell carcinoma, $^{10}$ colorectal cancer, ${ }^{11}$ breast cancer. $^{12}$ LINC00460 has been screened to be associated with the overall survival of ccRCC patients, but its prognostic value and effect on cellular function in ccRCC have not been studied.

As a master regulator of multiple protein-coding genes, lncRNAs can act as molecular bait, sponging and sequestering microRNAs (miRNAs), thereby inhibiting the interaction between miRNAs their target messenger RNAs (mRNA). ${ }^{6,7}$ In this way, lncRNAs act as competing endogenous, sponges with miRNAs, afterward regulate mRNAs and then regulate quite a lot of biological processes. ${ }^{8}$ Since this correlation among molecules is so close, abnormal expression of lncRNAs interferes with miRNA expression level led to a dysregulation that can promote or inhibit cancer development. ${ }^{9}$ So, the regulatory network of LINC00460-miRNA in ccRCC is an issue worth research.

In this research, we accessed the expression level of LINC00460 both in ccRCC tumor tissues and normal tissues, as well as in ccRCC cell lines and normal cell lines. The ability of LINC00460 as a prognostic factor for ccRCC was explored furthermore. Furthermore, the regulatory function of the LINC00460/miR-149-5p axis was investigated.

\section{Patients and Methods}

\section{Patients and Tissues Collection}

A total of 113 patients were enrolled in this study. The inclusion criteria were as follows: 1) patients with a histological diagnosis of ccRCC; (2) ccRCC patients underwent radical nephrectomy or partial nephrectomy, (3) ccRCC patients that did not receive any cancerrelated therapy, neither chemotherapy nor radiotherapy, nor targeted therapy; (4) patients with explicit clinical prognostic information. All patients have carried out the surgical operation for ccRCC tissues and an equal amount of normal paracancerous tissues between January 2011 and
March 2014. According to Chinese Clinical Practice Guidelines in Oncology (2010), the ccRCC patients with distant metastases did not meet the surgical indications, so there were no tissues obtained from patients with distant metastases. All tissues were quickly frozen and kept in liquid nitrogen before RNA extraction. The clinical baseline characteristics of the included ccRCC patients are described in Table 1. The follow-up lasted for 5 years, and survival information was recorded.

All participants gave their written informed consent when enrolled. The study was approved by the Ethics Committee of The Second Affiliated Hospital of Mudanjiang Medical University and also conducted following the Declaration of Helsinki.

\section{Cell Culture and Transient Transfection}

Human renal carcinoma cell lines 786-O, A-498, Caki-1, 769-P, and normal renal epithelial cells (HK-2) were all purchased from American Type Culture Collection (ATCC, Manassas, USA). Given the outstanding

Table I Clinical Characteristics of ccRCC Patients and Their Association with LINC00460 Expression

\begin{tabular}{|c|c|c|c|c|}
\hline \multirow[t]{2}{*}{ Characteristics } & \multirow[t]{2}{*}{$\begin{array}{c}\text { Cases } \\
n=I 13\end{array}$} & \multicolumn{2}{|c|}{$\begin{array}{l}\text { LINC00460 } \\
\text { Expression }\end{array}$} & \multirow[t]{2}{*}{$P$ values } \\
\hline & & $\begin{array}{c}\text { Low } \\
(n=54)\end{array}$ & $\begin{array}{c}\text { High } \\
(n=59)\end{array}$ & \\
\hline \multicolumn{5}{|l|}{ Gender } \\
\hline Male & 72 & 34 & 38 & 0.873 \\
\hline Female & 41 & 20 & 21 & \\
\hline \multicolumn{5}{|l|}{ Age } \\
\hline$\leq 55$ & 59 & 30 & 29 & 0.496 \\
\hline$>55$ & 54 & 24 & 30 & \\
\hline \multicolumn{5}{|l|}{ Tumor size $(\mathrm{cm})$} \\
\hline$\leq 5$ & 63 & 32 & 31 & 0.473 \\
\hline$>5$ & 50 & 22 & 28 & \\
\hline \multicolumn{5}{|l|}{$\begin{array}{l}\text { Lymph node } \\
\text { metastasis }\end{array}$} \\
\hline Negative & 79 & 44 & 35 & 0.010 \\
\hline Positive & 34 & 10 & 24 & \\
\hline \multicolumn{5}{|l|}{ Histological grade } \\
\hline I-II & 86 & 41 & 45 & 0.966 \\
\hline III-IV & 27 & 13 & 14 & \\
\hline \multicolumn{5}{|l|}{ TNM stage } \\
\hline I-II & 76 & 42 & 34 & 0.023 \\
\hline III-IV & 37 & 12 & 25 & \\
\hline
\end{tabular}

Note: Differences were analyzed using Pearson's chi-square test. 
expression of LINC00460, 786-O and/or Caki-1 cells were used for further experiments. 786-O, ACHN, Caki-1, and 769-P cells were all cultured in RPMI-1640 medium (HyClone, Logan, UT, USA) supplemented with 10\% FBS (HyClone). HK-2 cells were grown in keratinocyte serum-free medium (K-SFM; Invitrogen, USA) supplemented with $10 \%$ FBS (HyClone). All cells were routinely cultured in a humidified circumstance with $5 \% \quad \mathrm{CO}_{2}$ at $37^{\circ} \mathrm{C}$.

Small interfering RNAs (siRNAs) designed for LINC00460 (si-LINC00460) and siRNA negative control (si-NC) were obtained from RiboBio Co., Ltd. (Guangzhou, Guangdong, China), as well as the wildtype LINC00460 (WT-LINC00460) and mutant LINC00460 (MUT-LINC00460) and the corresponding negative control (anti-miR-NC). The Recombinant plasmid(s) were then transfected into 786-O cells or Caki-1 cells in the use of Lipofectamine 3000 (Invitrogen, Thermo Fisher Scientific, Inc., Waltham, MA, USA).

\section{RNA Isolation and Quantitative Real-Time Polymerase Chain Reaction (qRT-PCR) for RNA Expression Level}

Tissue specimens were homogenized in TRIzol reagent (Thermo Fisher Scientific, Inc.), and total RNA was isolated using the Qiagen RNeasy Plus Mini Kit (Qiagen, Venlo, The Netherlands) according to the protocol supplied by the manufacturer. The cells were directly treated with TRIzol reagent to extract total RNA. PrimeScript RT Master Mix (Takara Bio Inc.) was introduced to react with total RNA for synthesizing cDNA, while PrimeScript Reverse Transcriptase (Takara Bio Inc.) was used in the transcription from miRNAs to cDNA. Specific primers and SYBR $^{\circledR}$ Premix Ex Taq ${ }^{\mathrm{TM}}$ II (Takara Bio Inc.) participated in the reaction. The determination was done at a Thermal Cycler Dice ${ }^{\mathrm{TM}}$ Real-Time System Ш (Takara Bio Inc.). The relative expression was analyzed $2^{-\Delta \Delta \mathrm{Ct}}$ methods and normalized to that of GAPDH for LINC00460 while U6 for miR-149-5p.

\section{Cell Counting Kit (CCK)-8 Assay for Cell Proliferation}

The proliferation of ccRCC cells was determined by CCK8 assay. The transfected cells with a concentration of $5 \times 10^{3}$ cells/well were inoculated onto a 96 -well plate. And then, the cells were grown in a humidified incubator at $37^{\circ} \mathrm{C}$ with $5 \% \mathrm{CO}_{2} .10 \mu \mathrm{L}$ CCK-8 solution was added into each well at 24,48 , and $72 \mathrm{~h}$ of culture. After 2 $\mathrm{h}$ incubation, the values of absorbance were detected at $450 \mathrm{~nm}$ by the SpectraMax Plus 384 Microplate Reader (Molecular Devices, LLC).

\section{Transwell Assay for Cell Migration and Invasion}

Transwell assays served for the cell migration and invasion accession. Briefly, for the invasion assay, $2 \times 10^{4}$ transfected cells prepared in serum-free culture medium were reseeded into the top chamber (Corning Inc., Corning, NY, USA) with $300 \mu \mathrm{g} / \mathrm{mL}$ Matrigel (BD Bioscience, USA) while blank medium supplemented with $10 \%$ FBS was loaded into the lower well. After $24 \mathrm{~h}$ incubation at $37^{\circ} \mathrm{C}$, invasive cells that passed through the filter were fixed and stained with $0.1 \%$ crystal violet solution (ab246820, Abcam, Cambridge, USA). Cell count assay was completed in five random fields of the microscope vision. Concerning the migration assay, the test process was similar, but without the incubation of Matrigel in the top chamber.

\section{Luciferase Reporter Assay}

$1 \times 10^{5} 786-\mathrm{O}$ per well were implanted in 24 -well plates and co-transfected miR-149-5p or negative control (miR-NC) with WT-LINC00460 or MUT-LINC00460, which all purchased from RiboBio Co., Ltd. (Guangzhou, Guangdong, China). 48 hours after the transfection via Lipofectamine 2000 , the luciferase activity of firefly was detected at SpectraMax Gemini XPS (Molecular Devices, USA). The firefly luciferase activity ratio normalized to renilla luciferase activity was used to assess the binding relationship of LINC00460 and miR-149-5p.

\section{RNA Immunoprecipitation (RIP) Assay}

Magna RIPTM RNA-Binding Protein Immunoprecipitation Kit (Millipore, Billerica, MA, US) was used following the manufacturer's guide. RNA was extracted using the Total RNA Isolation Kit (Invitrogen, Carlsbad, USA), and qRTPCR was performed then. Ago-2 and IgG antibodies were obtained from Abcam (Cambridge, MA, USA).

\section{Statistical Analyses}

The statistical analyses were performed by SPSS 20.0 (SPSS Inc., Chicago, IL, USA) or GraphPad 5.0 (GraphPad Software, Inc., La Jolla, CA, USA). Each data was obtained from at least three independent experiments. The quantitative 
data are formed as mean \pm standard deviation. The differences between the two groups were analyzed using the 2-tailed Student's $t$-test, while those between multiple groups were analyzed using one-way analysis of variance (ANOVA) accompanied by Tukey's test. The correlation between LINC00460 expression and clinical parameters of ccRCC patients was examined via Pearson's chi-square test. The correlation between LINC00460 and miRNA-149-5p expression was tested by Spearman correlation analysis. The differences $P<0.05$ were considered statistically significant.

\section{Results}

\section{LINC00460 is Overexpressed in ccRCC} Tissues and Cell Lines

To determine the expression levels of LINC00460, 113 paired tumors and normal tissues were examined by RTqPCR. Higher expression of LINC00460 was observed in ccRCC tissues by comparison within normal tissue (Figure 1A). Determination was also done between ccRCC cells and normal renal cells, and the differential expression was obtained, too. As shown in Figure 1B, LINC00460 expression increased in different degrees and significantly in four ccRCC cells compared with normal kidney cells.

\section{Correlation Between Clinical Characteristics and LINC00460 \\ Expression in ccRCC Patients}

According to the median expression level of LINC00460 in ccRCC tissues, patients involved were divided into two groups: a low expression group and a high expression group. Then, the ccRCC patients' clinical baseline data were analyzed using the chi-square test (Table 1). The results showed that LINC00460 expression showed a significant correlation with lymph node metastasis and TNM stage ( $P=0.010$ and $P=0.023$, respectively), but not with other characteristics, for example, gender, age, tumor size $(P>0.05)$.

\section{Upregulation of LINC00460 is Associated with Poor Prognosis in ccRCC Patients}

For further proof of LINC00460 prognostic value in ccRCC, Kaplan-Meier survival curves accompanied by Log rank tests and multivariate Cox regression analyses were carried out. The analysis result demonstrated that ccRCC patients with a high expression level of LINC00460 presented a significantly worse overall survival by comparison with patients with a low expression level (Log rank test $P=0.014$, Figure 2). Multivariate analysis also supported that LINC00460 overexpression was a significant factor associated with overall survival and prognosis of ccRCC $(\mathrm{HR}=2.619,95 \% \mathrm{CI}: 1.110$ $6.178, P=0.028$ ) (Table 2).

\section{Upregulation of LINC00460 Promotes Proliferation, Migration, and Invasion of ccRCC Cells}

A worse survival outcome in patients with LINC00460 overexpression suggested LINC00460 may play an important role in the process of ccRCC. 786-O and Caki-1 cells, with a particular overexpression of LINC00460, were transfected with si-LINC00460 or si$\mathrm{NC}$ and the transfection effect was verified via LINC00460 expression levels $(P<0.001$, Figure 3A and $\mathrm{B})$. CCK-8 assay was adopted to access the proliferative ability of ccRCC cells, and the result showed cell proliferation was significantly suppressed by
A

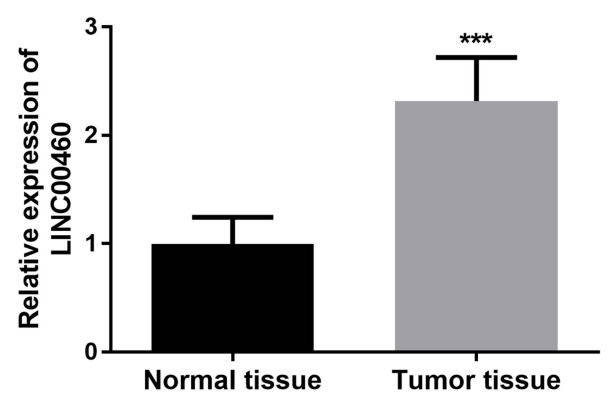

B

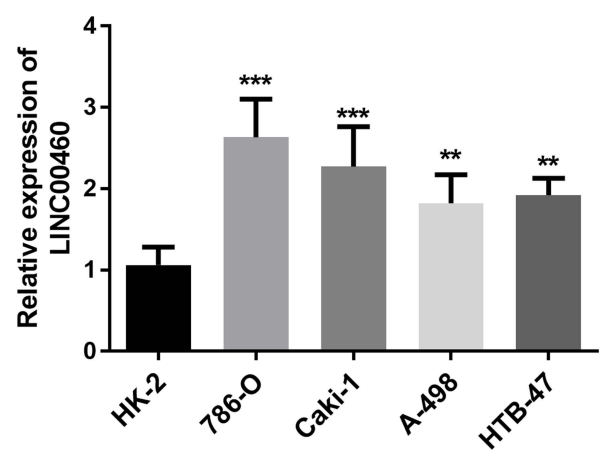

Figure I Relative LINC00460 expression in ccRCC tissues and cell lines. (A) The qRT-PCR analysis detected the expression level of LINC00460 in the RCC tissues and the corresponding normal tissues. (B) qRT-PCR analysis of LINC00460 in ccRCC cell lines and normal renal epithelial HK-2 cells. $* * P<0.0 \mathrm{I}$, $* * * P<0.00 \mathrm{I}$. 


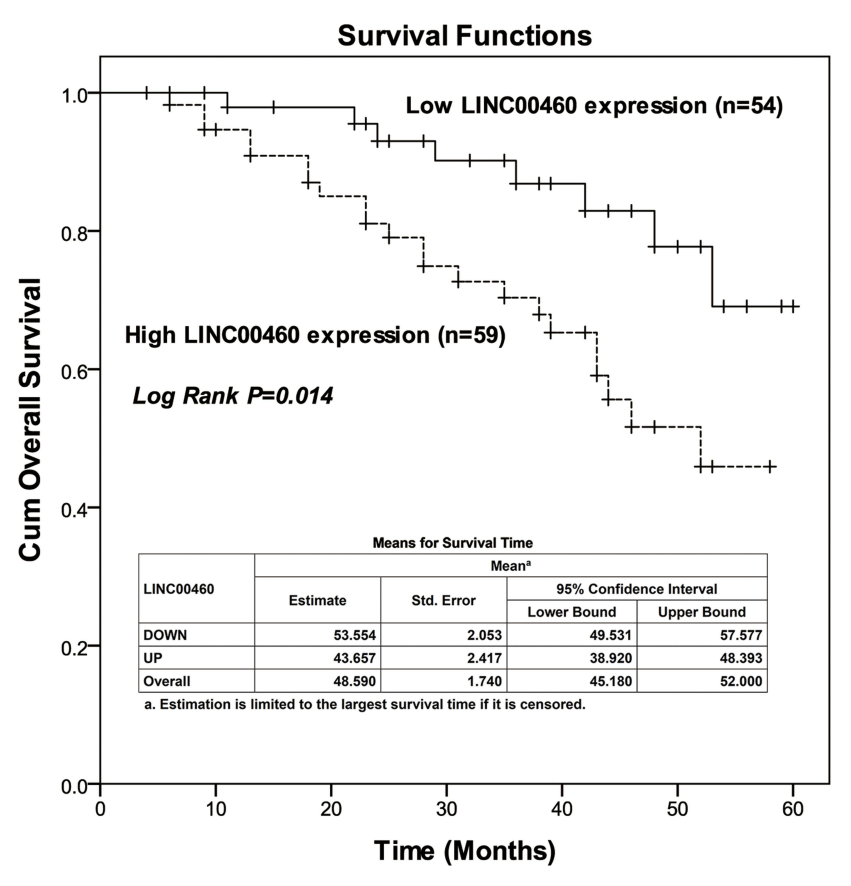

Figure 2 Kaplan-Meier curves for ccRCC patients with high and low levels of LINC00460 (Log rank test: $P=0.014)$.

LINC00460 knockdown $(P<0.001$, Figure 3C and D). The migration and invasive ability of 786-O and Caki1 cells were significantly inhibited compared with that of untreated cells and cells transfected si-NC $(P<0.001$, Figure $4 \mathrm{~A}-\mathrm{D})$ via the transwell assays.

\section{MiR-149-5p Acted as the Target of LINC00460}

To clarify the potential mechanism, bioinformatics (lncRNASNP2 database, http://bioinfo.life.hust.edu.cn/) was used to predict the potential target miRNA of LINC00460. miR-149-5p was predicted to have binding sites with LINC00460 (Figure 5A). Next, miR-149-5p

Table 2 Multivariate Cox Analyses of Overall Survival of ccRCC Patients

\begin{tabular}{|l|c|c|c|}
\hline \multirow{2}{*}{ Characteristics } & \multicolumn{3}{|c|}{ Multivariate Cox Analysis } \\
\cline { 2 - 4 } & HR & $\mathbf{9 5 \%} \mathbf{~ C ~}$ & P value \\
\hline LINC00460 & 2.619 & $1.110-6.178$ & 0.028 \\
Gender & 1.450 & $0.645-3.259$ & 0.369 \\
Age & 1.835 & $0.842-3.998$ & 0.127 \\
Tumor size & 1.423 & $0.647-3.131$ & $0.38 \mathrm{I}$ \\
Lymph node metastasis & 2.467 & $1.088-5.591$ & $0.03 \mathrm{I}$ \\
Histological grade & 1.614 & $0.627-4.155$ & $0.32 \mathrm{I}$ \\
TNM stage & 2.577 & $1.039-6.391$ & $0.04 \mathrm{I}$ \\
\hline
\end{tabular}

Note: Data are analyzed using multivariate Cox regression analysis. was found a significant downregulation in ccRCC tissues and cells compared with that in non-tumorous renal tissues or normal renal cells (Figure 5B and $\mathrm{C}$ ). Additionally, LINC00460 expression was negatively related to miR-149-5p expression in ccRCC tissues revealed by Spearman correlation analysis (Figure 5D). According to the binding sites between LINC00460 and miR-149-5p, MUT-LINC00460 was obtained. The 786-O cells were co-transfected with recombinant luciferase reporter plasmid of wildtype LINC00460 and mutated one respectively and control miRNA or miRNA-149-5p. From the result of luciferase activity, significant inhibition was revealed in the WT-LINC00460 transfected cells when miR-149-5p was overexpressed, but no influence was found in the mutated MUT-LINC00460 transfected cells (Figure 5E). Furthermore, LINC00460 and miR149-5p both had the disposition to enrich in Ago2 in 786-O cells (Figure 5F).

\section{Discussion}

There is growing evidence that lncRNA expression level is associated with prognostic outcome in many cancers. ${ }^{13-16}$ A previous study indicated that LncRNAIL7R correlated to poor outcomes in cervical cancer patients and is an independent prognostic factor for cervical cancer. ${ }^{17}$ Zheng et al used lncRNA microarray assay to identify gastric cancer (GC)-specific exosomal lncRNAs and found one particular lncRNA, lncSLC2A12-10:1, might be a potential prognosis monitoring factor for GC. ${ }^{18}$ Moreover, Lnc-IRF2-3 and Lnc-ZNF667-AS1 owned high specificity and sensitivity to distinguish B-chronic lymphocytic leukemia (B-CLL) between healthy controls and were potential prognostic indicators in B-CLL. ${ }^{19}$ For RCC, quite a few lncRNAs have been identified to have involvement in diagnosis or prognosis. ${ }^{20}$ Ellinger et al quantified the lnc-ZNF180-2 expression in patients with ccRCC and concluded it may be a useful prediction factor of ccRCC patients' outcomes.

In this study, the ability of LINC00460 as a prognosis indicator was accessed by the expression level, chi-square test, Kaplan-Meier survival curves, and multivariate Cox regression analyses. A significantly upregulated expression of LINC00460 was found both in ccRCC tissue and cells compared with normal tissues and cells. LINC00460 expression levels allowed the distinction of ccRCC and normal renal tissue or cells. Then, the high expression of 


\section{A}
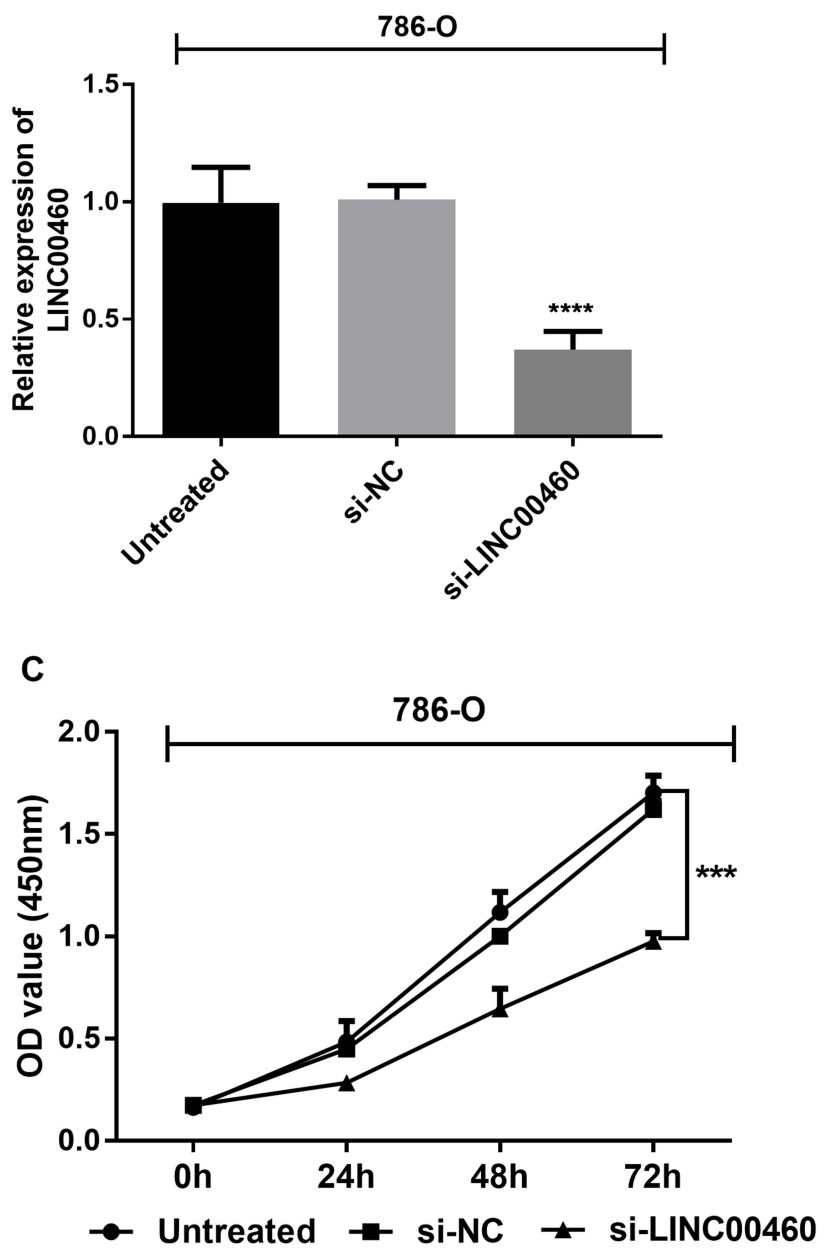

B

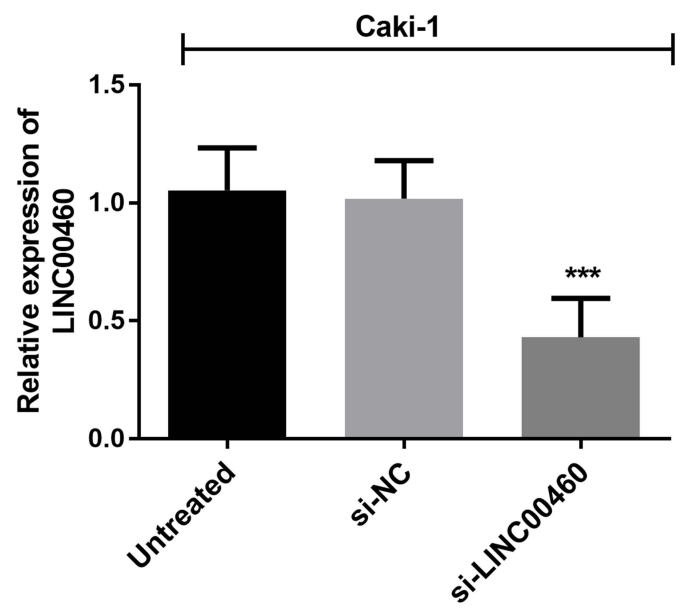

D

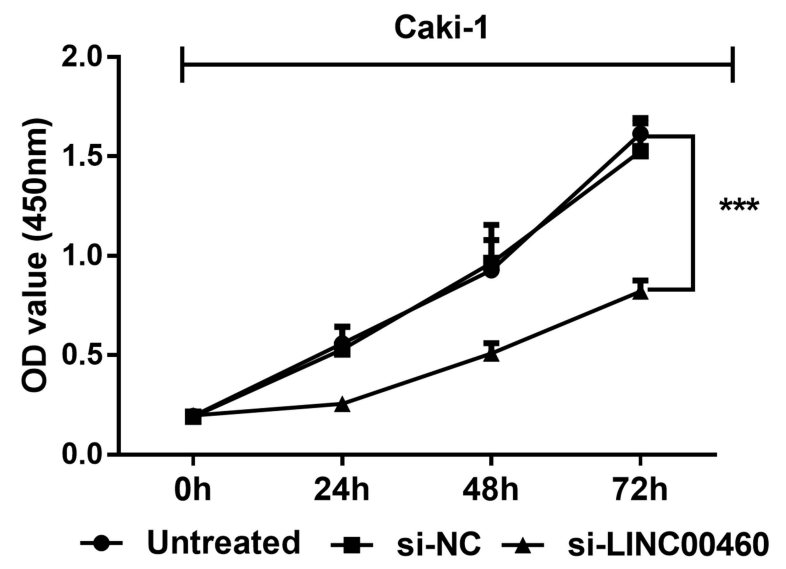

Figure 3 Knockdown of LINC00460 inhibited the proliferation, migration and invasion of 786-O and Caki-I cells. (A and B) si-NC or si-LINC00460 were transfected into 786-O and Caki-I cells, and transfection efficiency was verified by qRT-PCR. (C and D) Cell proliferation of 786-O and Caki-I was determined after transfection with si-NC or si-LINC00460 by CCK-8 assays. ***P or *****P $<0.001$.

LINC00460 was found to be markedly associated with lymph node metastasis and TNM stages. So, enhanced LINC00460 expression was relevant to the poor prognosis of RCC patients. Besides, a promotive effect of increased LINC00460 was found on proliferation, migration, and invasion of ccRCC cells, and this result coincided with that of Zhang et al. ${ }^{21}$ Consequently, LINC00460 can be a promising prognostic biomarker, even a therapeutic target for patients suffering from ccRCC.

Recent works have shown that LINC00460 exerted an accelerating or inhibitory effect on the biological activities of different types of cancer cells. ${ }^{22,23}$ For instance, LINC00460 is involved in the process of gastric cancer and its inhibition would suppress cell proliferation, invasion, and $\mathrm{S}$ phase cell number of gastric cancer cells. ${ }^{24}$ The latest study reported that down-regulation of LINC00460 can potentially suppress proliferation, invasion, migration of colorectal cancer cells. ${ }^{25}$ Given these, we tried to explore the impact of LINC00460 expression on ccRCC cells. 786-O and Caki-1 cells were transfected with small interfering RNA for LINC00460 and corresponding blank. By CCK-8 assay and transwell assay, the potentiality of proliferation, migration, and invasion in ccRCC cells exhibited inhibitory when LINC00460 was knocked down. This is consistent with the role of LINC00460 in other tumor cells.

The biological significance of LINC00460 in ccRCC cells suggested that this RNA functioned as a ceRNA decoy for some tumor-suppressive miRNAs. To find out the possible target miRNA, bioinformatics was applied and lncRNASNP2 database predicted that miR-149-5p was a downstream target miRNA of 

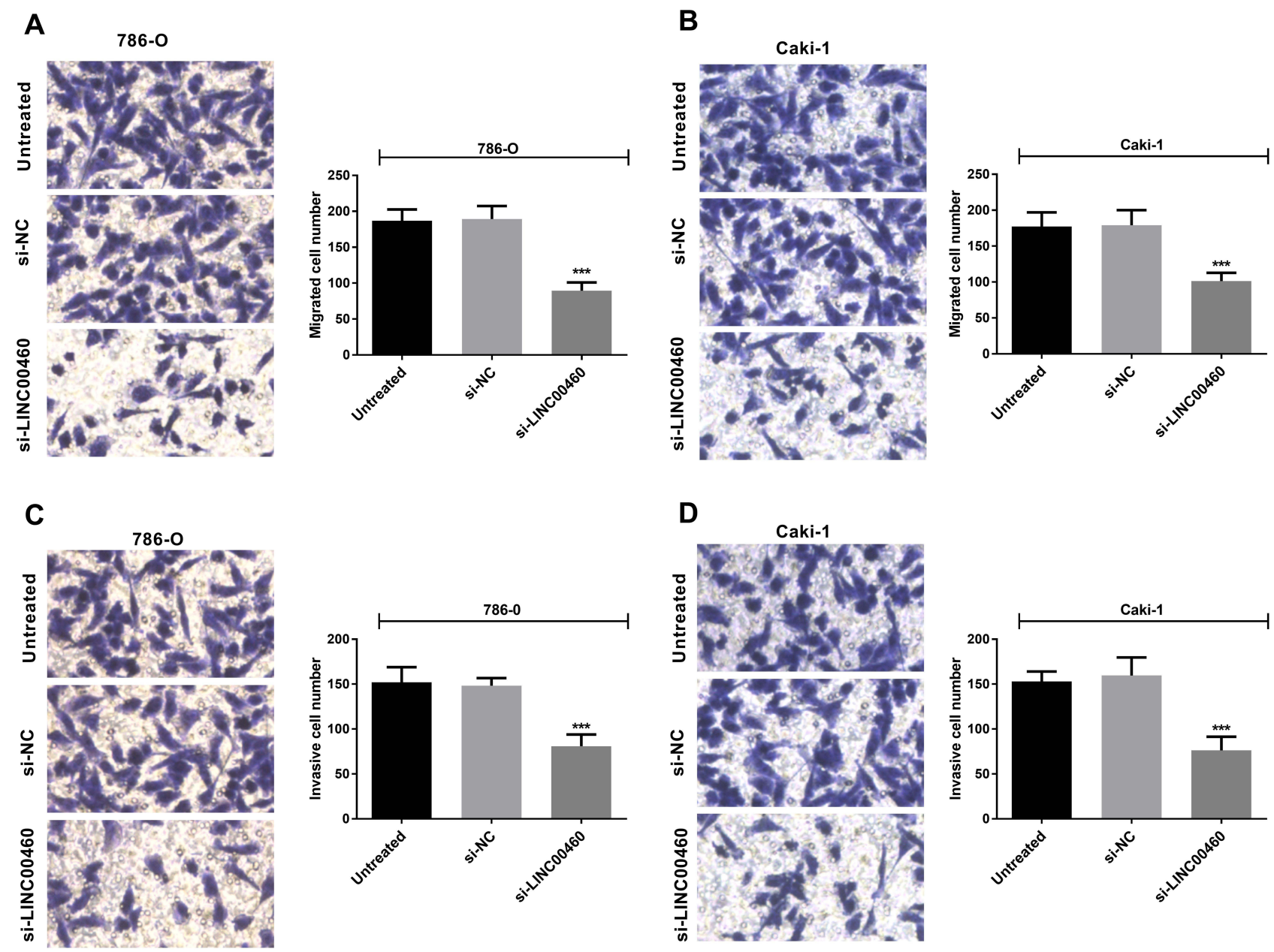

Figure 4 (A and B) The migration abilities of 786-O and Caki-I cells were accessed after transfection via transwell migration assays. (C and D) The invasive abilities of 786-O and Caki-I cells were tested after transfection using transwell invasion assays. $* * * P<0.001$.

LINC00460. Interestingly, miR-149-5p was downregulated in ccRCC tissues, correlated with the survival of patients with ccRCC, ${ }^{26}$ and acted as a tumor suppressor in $\mathrm{RCC}^{27}$ Down expression of miR-149-5p in ccRCC tissue and cells reflected the sponge effect from LINC00460, and luciferase reporter assays along with RIP assay directly verified that miR-1495p 3'-UTR was the target of LINC00460. miR-149-5p, as a tumor suppressor, can be sponged by various lncRNAs, such as LncRNA MIAT, ${ }^{28}$ LncRNA DRAIC, ${ }^{29}$ IncRNA SNHG8 ${ }^{30}$ which play key roles in normal and cancerous tissues. Moreover, forkhead box protein M1 (FOXM1) was reported to be directly regulated by miR-149-5p in ccRCC cells. ${ }^{31}$ Combining these previous studies and our present results, it is speculated that LINC00460 might promote ccRCC cell proliferation, migration, and invasion by sponging
miR-149-5p and then regulating FOXM1. The significance of the LINC00460/miR-149-5p/FOXM1 axis in ccRCC will be the next research point.

\section{Conclusion}

In conclusion, the findings in this study showed that LINC00460 expression was upregulated in human ccRCC tissues and cell lines, and this upregulation is associated with a poor prognosis. Downregulated LINC00460 could suppress the proliferation, migration, and invasion of ccRCC cells, might via sponging miR-149-5p. This study suggests LINC00460 can act as a prognostic predictor and a molecular therapy target of ccRCC. What is more, these findings may lead to the development of personalized treatments for ccRCC. 
A

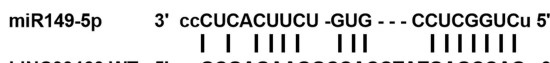
LINC00460 WT 5' caGCGAGAAGGCCACCTATGAGCCAGa LINC00460 MUT 5' caCCCACUUCCCGUGCTATCUCGGUCa 3 '
D

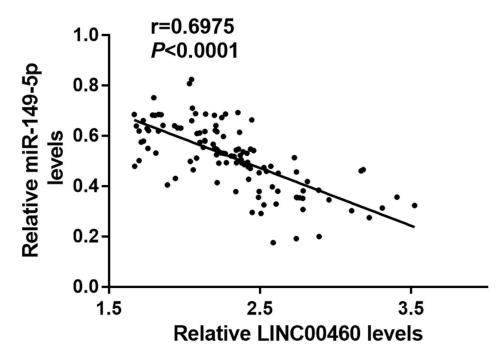

B

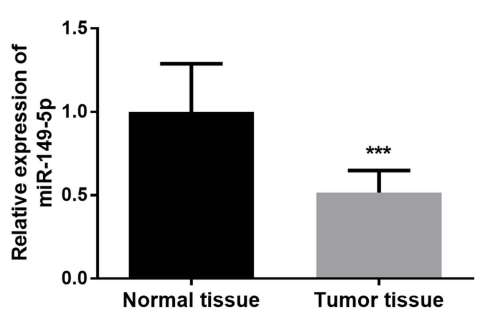

E

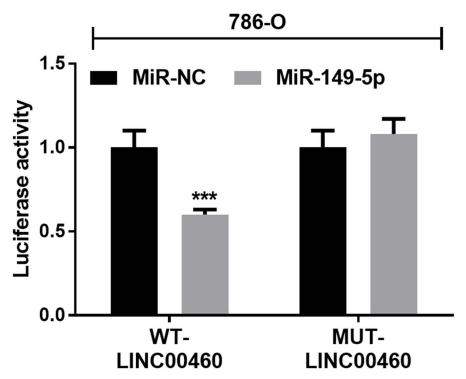

C

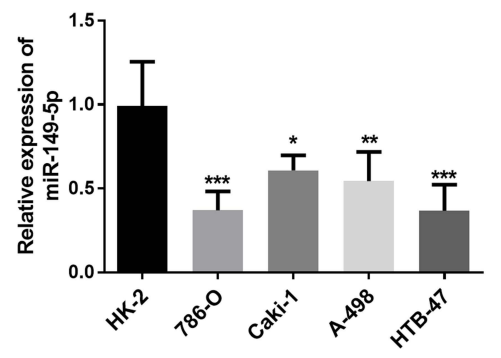

F

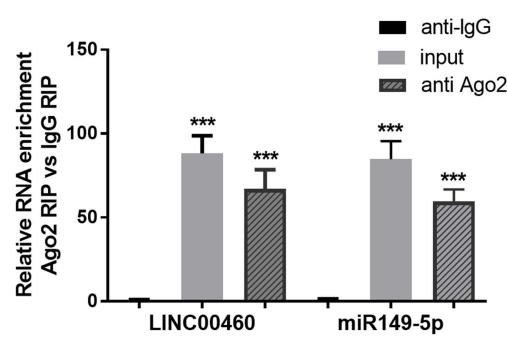

Figure 5 MiR-149-5p acted as the target of LINC00460. (A) The binding sites between LINC00460 and miR-I49-5p. (B) The qRT-PCR analysis detected the expression level of miR-149-5p in the RCC tissues and the corresponding normal tissues. (C) qRT-PCR analysis of miR-I49-5p in ccRCC cell lines and normal renal epithelial HK-2 cells. (D) The correlation between LINC00460 and miR-149-5p in ccRCC tissues was analyzed using Spearman correlation analysis. (E) Luciferase activity was examined in ccRCC cells co-transfected with WT-LINC00460 or MUT-LINC00460 reporter plasmid and miR-I49-5p or miR-NC. (F) RIP assay indicated that both LINC00460 and miR-I49-5p were significantly enriched in Ago2 immunoprecipitate. Empty vector was used as a nonspecific control. IgG acted as an internal control. $* P<0.05$, $* * P<0.01, * * * P<0.001$.

\section{Ethics Statement}

All participants gave their written informed consent when enrolled. The study was approved by the Ethics Committee of The Second Affiliated Hospital of Mudanjiang Medical University and also conducted following the Declaration of Helsinki.

\section{Disclosure}

The authors report no conflicts of interest in this work.

\section{References}

1. Padala SA, Kallam A. Clear cell renal carcinoma. In: StatPearls. StatPearls Publishing LLC; 2020.

2. Capitanio U, Montorsi F. Renal cancer. Lancet. 2016;387 (10021):894-906. doi:10.1016/S0140-6736(15)00046-X

3. Arora RD, Limaiem F. Renal clear cell cancer. In: StatPearls. StatPearls Publishing LLC; 2020.

4. Matsuda T, Hori M. Five-year relative survival rate of kidney and renal pelvis cancer in the USA, Europe and Japan. Jpn J Clin Oncol. 2015;45(1):136. doi:10.1093/jjco/hyu216

5. Iyer MK, Niknafs YS, Malik R, et al. The landscape of long noncoding RNAs in the human transcriptome. Nat Genet. 2015;47(3):199-208. doi:10.1038/ng.3192

6. Cabili MN, Trapnell C, Goff L, et al. Integrative annotation of human large intergenic noncoding RNAs reveals global properties and specific subclasses. Genes Dev. 2011;25(18):1915-1927. doi:10.1101/ gad. 17446611
7. Shima H, Kida K, Adachi S, et al. Lnc RNA H19 is associated with poor prognosis in breast cancer patients and promotes cancer stemness. Breast Cancer Res Treat. 2018;170(3):507-516. doi:10.1007/s10549-018-4793-z

8. Wang Y, Ding X, Hu H, et al. Long non-coding RNA lnc-PCTST predicts prognosis through inhibiting progression of pancreatic cancer by downregulation of TACC-3. Int $J$ Cancer. 2018;143 (12):3143-3154. doi:10.1002/ijc.31657

9. Angenard G, Merdrignac A, Louis C, Edeline J, Coulouarn C. Expression of long non-coding RNA ANRIL predicts a poor prognosis in intrahepatic cholangiocarcinoma. Dig Liver Dis. 2019;51 (9):1337-1343. doi:10.1016/j.dld.2019.03.019

10. Qi L, Zhang T, Yao Y, et al. Identification of lncRNAs associated with lung squamous cell carcinoma prognosis in the competitive endogenous RNA network. PeerJ. 2019;7:e7727. doi:10.7717/peerj.7727

11. Zhang Y, Liu X, Li Q, Zhang Y. IncRNA LINC00460 promoted colorectal cancer cells metastasis via miR-939-5p sponging. Cancer Manag Res. 2019;11:1779-1789. doi:10.2147/CMAR.S192452

12. Zhu Y, Yang L, Chong QY, et al. Long noncoding RNA Linc00460 promotes breast cancer progression by regulating the miR-489-5p/ FGF7/AKT axis. Cancer Manag Res. 2019;11:5983-6001. doi:10.2147/CMAR.S207084

13. Song H, He P, Shao T, Li Y, Li J, Zhang Y. Long non-coding RNA XIST functions as an oncogene in human colorectal cancer by targeting miR-132-3p. J buon. 2017;22(3):696-703.

14. Wei $\mathrm{H}, \mathrm{Hu} \mathrm{J}, \mathrm{Pu} \mathrm{J}$, et al. Long noncoding RNA HAGLROS promotes cell proliferation, inhibits apoptosis and enhances autophagy via regulating miR-5095/ATG12 axis in hepatocellular carcinoma cells. Int Immunopharmacol. 2019;73:72-80. doi:10.1016/j.intimp.2019.04.049

15. Da M, Ma J, Zhang Y, et al. High expression level of long non-coding RNA HOTAIR is associated with poor overall survival in gastric cancer patients: evidence from meta-analysis. $J$ buon. 2017;22;4:911-918 
16. Jiang CY, Gao Y, Wang XJ, et al. Long non-coding RNA lnc-MX1-1 is associated with poor clinical features and promotes cellular proliferation and invasiveness in prostate cancer. Biochem Biophys Res Commun. 2016;470(3):721-727. doi:10.1016/j.bbrc.2016.01.056

17. Fan Y, Nan Y, Huang J, Zhong H, Zhou W. Up-regulation of inflammation-related LncRNA-IL7R predicts poor clinical outcome in patients with cervical cancer. Biosci Rep. 2018;38(3).

18. Zheng $\mathrm{P}$, Zhang $\mathrm{H}$, Gao $\mathrm{H}$, et al. Plasma exosomal long noncoding RNA lnc-SLC2A12-10:1 as a novel diagnostic biomarker for gastric cancer. Onco Targets Ther. 2020;13:4009-4018. doi:10.2147/OTT. S253600

19. El-Khazragy N, Esmaiel MA, Mohamed MM, Hassan NS. Upregulation of long noncoding RNA Lnc-IRF2-3 and Lnc-ZNF667-AS1 is associated with poor survival in B-chronic lymphocytic leukemia. Int J Lab Hematol. 2020;42(3):284-291. doi:10.1111/ijlh.13167

20. Blondeau JJ, Deng M, Syring I, et al. Identification of novel long non-coding RNAs in clear cell renal cell carcinoma. Clin Epigenetics. 2015;7(1):10. doi:10.1186/s13148-015-0047-7

21. Zhang D, Zeng S, Hu X. Identification of a three-long noncoding RNA prognostic model involved competitive endogenous RNA in kidney renal clear cell carcinoma. Cancer Cell Int. 2020;20(1):319. doi:10.1186/s12935-020-01423-4

22. $\mathrm{Hu}$ X, Liu W, Jiang X, et al. Long noncoding RNA LINC00460 aggravates invasion and metastasis by targeting miR-30a-3p/Rap $1 \mathrm{~A}$ in nasopharyngeal carcinoma. Hum Cell. 2019;32(4):465-476. doi:10.1007/s13577-019-00262-4

23. Wang HX, Kang LJ, Qin X, Xu J, Fei JW. LINC00460 promotes proliferation and inhibits apoptosis of non-small cell lung cancer cells through targeted regulation of miR-539. Eur Rev Med Pharmacol Sci. 2020;24(12):6752-6758.

24. Zhang S, Xu J, Wang H, Guo H. Downregulation of long noncoding RNA LINC00460 expression suppresses tumor growth in vitro and in vivo in gastric cancer. Cancer Biomark. 2019;24(4):429-437. doi:10.3233/CBM-182177
25. Yuan B, Yang J, Gu H, Ma C. Down-regulation of LINC00460 represses metastasis of colorectal cancer via WWC2. Dig Dis Sci. 2020;65(2):442-456. doi:10.1007/s10620-019-05801-5

26. Xie M, Lv Y, Liu Z, et al. Identification and validation of a four-miRNA (miRNA-21-5p, miRNA-9-5p, miR-149-5p, and miRNA-30b-5p) prognosis signature in clear cell renal cell carcinoma. Cancer Manag Res. 2018;10:5759-5766. doi:10.2147/ CMAR.S187109

27. Jin L, Li Y, Liu J, et al. Tumor suppressor miR-149-5p is associated with cellular migration, proliferation and apoptosis in renal cell carcinoma. Mol Med Rep. 2016;13(6):5386-5392. doi:10.3892/ mmr.2016.5205

28. Ye ZM, Yang S, Xia YP, et al. LncRNA MIAT sponges miR-149-5p to inhibit efferocytosis in advanced atherosclerosis through CD47 upregulation. Cell Death Dis. 2019;10(2):138. doi:10.1038/s41419019-1409-4

29. Li F, Zhou X, Chen M, Fan W. Regulatory effect of LncRNA DRAIC/miR-149-5p/NFIB molecular network on autophagy of esophageal cancer cells and its biological behavior. Exp Mol Pathol. 2020;116:104491. doi:10.1016/j.yexmp.2020.104491

30. Dong J, Teng F, Guo W, Yang J, Ding G, Fu Z. IncRNA SNHG8 promotes the tumorigenesis and metastasis by sponging miR-149-5p and predicts tumor recurrence in hepatocellular carcinoma. Cell Physiol Biochem. 2018;51(5):2262-2274. doi:10.1159/000495871

31. Okato A, Arai T, Yamada Y, et al. Dual strands of pre-miR-149 inhibit cancer cell migration and invasion through targeting FOXM1 in renal cell carcinoma. Int J Mol Sci. 2017;18(9):1969.

\section{Publish your work in this journal}

Cancer Management and Research is an international, peer-reviewed open access journal focusing on cancer research and the optimal use of preventative and integrated treatment interventions to achieve improved outcomes, enhanced survival and quality of life for the cancer patient.
The manuscript management system is completely online and includes a very quick and fair peer-review system, which is all easy to use. Visit http://www.dovepress.com/testimonials.php to read real quotes from published authors. 